\title{
Dyssynchrony Assessment in Arrhythmogenic Cardiomyopathy With Left Ventricular Involvement
}

\author{
Yolanda Vives-Gilabert ${ }^{1}$, Jorge Sanz-Sánchez ${ }^{2}$, Antonio Cebrián ${ }^{1}$, Raquel Cervigón ${ }^{3}$, José Millet ${ }^{1}$, \\ Esther Zorio ${ }^{2}$, Francisco Castells ${ }^{1}$ \\ ${ }^{1}$ Istituto ITACA, Universitat Politècnica de València, Valencia, Spain \\ ${ }^{2}$ Hospital Universitari i Politècnic La Fe, Valencia, Spain \\ ${ }^{3}$ Universidad de Castilla-la Mancha, Cuenca, Spain
}

\begin{abstract}
Arrhythmogenic cardiomyopathy $(A C)$ is an inherited rare disease that can cause sudden cardiac death. Due to its heterogeneous phenotipe, a compendium of criteria collected in a Task Force Criteria (TFC) have to be met for its diagnosis, being dysshynchrony one of those criteria. The classical form of the disease involves the right ventricle, while the left ventricle $A C(A C-L V)$ has been less studied. We aim to study dyssynchrony in AC-LV patients.

The sample consisted of 36 subjects diagnosed with $A C$ $L V$ and 23 non-affected relatives who were non-carriers of the pathogenic mutation of the proband. From each individual, radial, circumferential and longitudinal strain were obtained in the 16 AHA segments of the myocardium and dyssynchrony was calculated as the standard deviation of the time-to-peak strain. LV ejection fraction (LVEF) was also obtained. Finally, a clustering algorithm was applied to the 3-axis dyssynchronies and the LVEF.

The clustering algorithm performed well (silhouette $=$ 0.6 ) and detected 2 clusters (cluster $1=23$ controls +19 $A C-L V$ patients and cluster $2=17$ AC- $L V$ patients). Radial dyssynchrony was the feature with the higher predictor importance.

In conclusion, $47 \%$ of AC- $L V$ patients present impaired dyssynchrony. Radial dyssynchrony was the most affected parameter among all dyssynchronies and LVEF.
\end{abstract}

\section{Introduction}

Arrythmogenic cardiomyopathy (AC) is an inherited heart muscle disorder that results in fibrofatty replacement of the ventricle and patients are at high risk of sudden cardiac death [1].

The "classical" subtype of this disease involves a predilection of the right ventricle, however in the recent years, new cases of left-dominant AC (AC-LV) have been characterized [2]. As the phenotype of this disease is very heterogeneous, its diagnosis becomes challenging. The diagnosis of the right ventricular $\mathrm{AC}$ is guided by a Task Force Criteria (TFC) [3], where a compendium of criteria have to be met, however, the diagnosis of the left dominant form of AC has not yet been contemplated in the TFC and it needs to be studied more in depth.

Cardiovascular imaging has helped in the recent years to improve diagnosis in AC, as reflected in the category "Global or regional dysfunction and structural alterations" of the 2010 TFC, which includes ejection fraction, akinesia/dyskinesia and dyssychronous contraction among others.

LV ejection fraction describes the volumetric fraction of blood ejected from the LV with each heartbeat and it represents a measure of the pumping efficiency of the left ventricle, an indicator of heart failure [4]. Akinesia/dyskinesia describes disorder in movement, that can be measured by strain, which is the deformation of the myocardium. Feature tracking algorithms are capable of quantifing strain time curves and extracting strain-related parameters like peak strain and time to peak strain among others [5]. From the time to peak strain it is possible to obtain the dyssynchronous contraction of the myocardium [6].

Some studies related to strain and dyssynchrony in right ventricular $\mathrm{AC}$ have been performed in the past [6,7], but as far as we know, we are one of the first groups to investigate strain and dyssynchrony in $\mathrm{AC}$ with $\mathrm{LV}$ involvement [8].

Taking into account the variable phenotype of this disease, the objective of this study is to analyse the percentage of AC-LV patients with dyssynchronous contraction and LV ejection fraction. For this purpose, clustering algorithms can be applied [9].

Clustering refers to the task of grouping a set of subjects by similarity according to their features. Consequently, 
AC-LV patients could be divided into clusters according to their degree of dyssynchrony and LV ejection fraction. With these algorithms, it is also possible to know which of the input features or parameters is the most discriminant between clusters.

\section{Materials and methods}

\subsection{Sample}

The sample was composed of 36 patients diagnosed with AC with left ventricular involvement and 23 nonaffected relatives who were non-carriers of the pathogenic mutation of the proband. Patients were diagnosed following 2010 TFC and the left ventricular predominance was determined applying the scoring system previously reported [10]. All clinical details of the sample have already been published before [8].

\subsection{MRI acquisition}

All cine CMR studies were acquired in a 1.5-Tesla scanner from different vendors (Siemens Avanto, Siemens Symphony and GE Signa HDxt). Steady-state free precession pulse sequence was used to acquire two-, threeand four-chamber long-axis slices plus contiguous shortaxis slices with 20-25 phases per cycle, 6 to $8 \mathrm{~mm}$ slice thickness, FOV $=360 \times 480$ and a matrix size of $196 \times 172$.

\subsection{Strain and dyssynchrony}

The feature tracking module of Circle (Circle CVI ${ }^{42}$ version 5.5.1, Calgary, Canada) was used to manually delineate the endocardium and the epicardium at enddiastole of the three long-axis and the short-axis slices and calculate radial, circumferential and longitudinal strainrelated parameters in the 16 AHA segments.

Strain curves represent the deformation of the myocardium in time in the three directions. Radial strain represents the wall thickening during systole and thinning during diastole. Circumferential and longitudinal strain values describe the shortening in systole and the lengthening in diastole, along the circular perimeters for the circumferential strain and along the longitudinal axis (from the base to the apex) for the longitudinal strain.

For each subject, we obtained the time to the maximum strain value for each of the 16 AHA segments. Dyssynchrony was then calculated as the standard deviation of the 16 time-to-peak values, as the example shown in Figure 1.

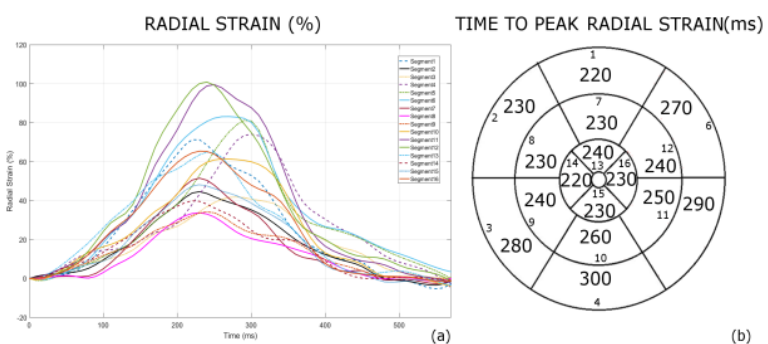

Figure 1. Radial strain time curves of the 16 AHA segments (a) and time to peak strain (ms) per segment (b).

\subsection{Left ventricle ejection fraction}

The left ventricle ejection fraction (LVEF) was calculated from the endocardial volume (V) time curves, also calculated by Circle.

$$
E F=\frac{\mathrm{V}\left(t_{D}\right)-V\left(t_{S}\right)}{V\left(t_{D}\right)}
$$

where $\mathrm{V}\left(\mathrm{t}_{\mathrm{D}}\right)=$ end-diastolic volume and $\mathrm{V}\left(\mathrm{t}_{\mathrm{S}}\right)=$ endsystolic volume [11].

\subsection{Clustering and statistical test}

To make a categorization of the dyssynchrony among patients we applied the SPSS Two-Step Cluster Component algorithm [12] of IBM SPSS (version 23, SPSS Statistics/IBM Corp, Chicago II, USA) to our sample according to four parameters: radial, circumferential and longitudinal dyssynchronies and LVEF.

In brief, this algorithm uses a sequential clustering approach to pre-cluster the data and then with an agglomerative hierarchical method make a final clustering of the results of the first step. It also detects automatically the number of clusters using Bayesian information and refining the result with distance values [13].

The silhouette method was applied to validate the consistency of the clusters [14].

Independent two-sample t-tests of each variable (dyssynchronies and LVEF) were also performed using SPSS software to test for significant differences between clusters. Bonferroni-corrected p-values were reported.

\section{Results}

The clustering algorithm obtained a good cluster quality with a silhouette value of 0.6 . The algorithm identified two clusters: Cluster 1 included 42 subjects, of which 23 were controls and 19 were AC-LV patients and Cluster2 included 17 AC-LV patients (without any controls) (Figure 2). 


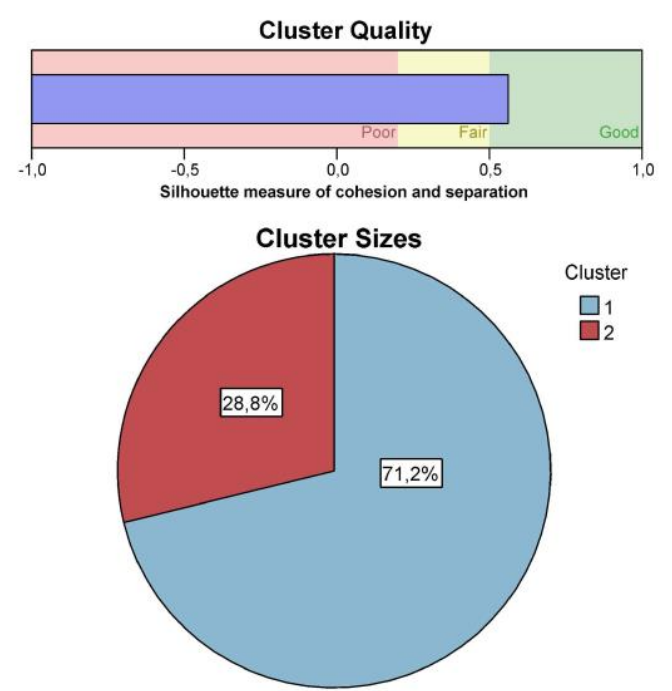

Figure 2. Clustering quality of the algorithm and distribution of the sample among clusters

For each cluster, mean values of the 4 parameters are shown in Table 1. We found significant differences between both clusters in all the parameters, with p-values $<0.001$.

Table 1 . Dyssynchrony and ejection fraction mean \pm std values per cluster.

\begin{tabular}{lll}
\hline Parameters & $\begin{array}{l}\text { Cluster 1 } \\
\text { (23 controls+ } \\
19 \mathrm{AC}-\mathrm{LV})\end{array}$ & $\begin{array}{l}\text { Cluster 2 } \\
(17 \mathrm{AC}-\mathrm{LV})\end{array}$ \\
\hline $\begin{array}{l}\text { Radial } \\
\text { dyssynchrony (ms) }\end{array}$ & $48.53 \pm 13.92$ & $90.75 \pm 21.27$ \\
$\begin{array}{l}\text { Circumferential } \\
\text { dyssynchrony (ms) }\end{array}$ & $39.61 \pm 8.72$ & $62.83 \pm 15.76$ \\
$\begin{array}{l}\text { Longitudinal } \\
\text { dyssynchrony (ms) }\end{array}$ & $45.34 \pm 10.64$ & $69.66 \pm 28.58$ \\
$\begin{array}{l}\text { LV ejection } \\
\text { fraction (\%) }\end{array}$ & $56.79 \pm 7.66$ & $40.71 \pm 12.06$ \\
\hline
\end{tabular}

Among the variables used for the clustering, radial dyssynchrony was the one with the higher predictor importance, followed by circumferential dyssynchrony and LVEF. The less discriminant variable was the longitudinal dyssynchrony (Figure 3).

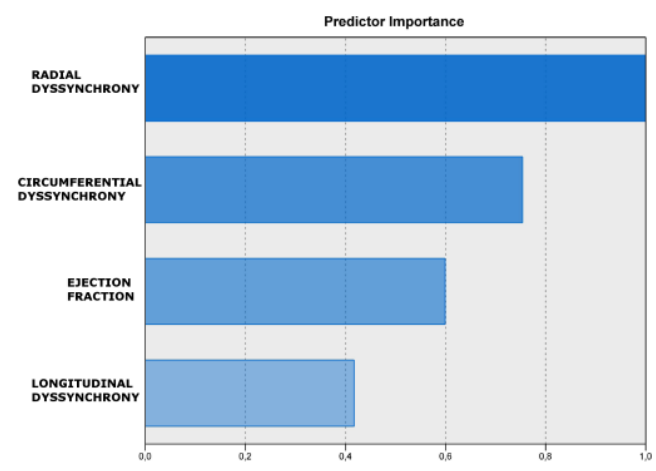

Figure 3. Predictor importance of dyssynchronies and LVEF

In Figure 4 we represent the distribution the radial dyssynchrony, that was the most discriminant variable.
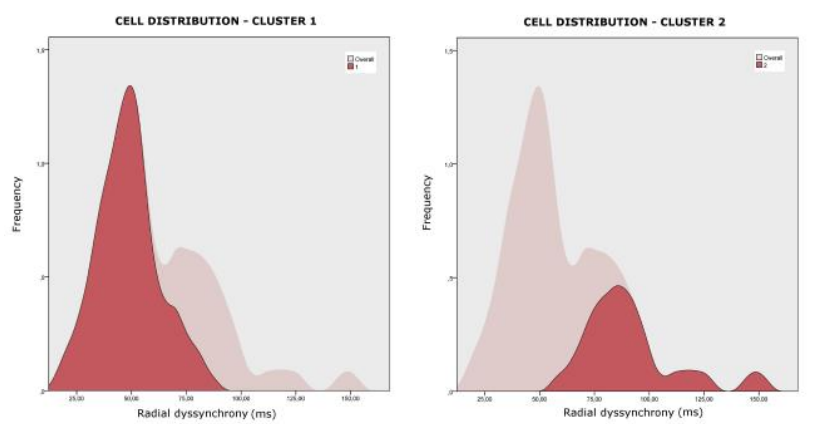

Figure 4. Frequency of radial dyssynchrony (ms) among clusters

\section{Discussion}

The diagnosis of AC-LV is challenging due to the heterogeneity of the disease and the lack of criteria for predominant left-ventricular AC.

The study herein presented describes the dyssynchrony and the ejection fraction in patients with $\mathrm{AC}$ with $\mathrm{LV}$ involvement. We found that: 1) $47 \%$ of the AC-LV patients (17 patients) presented dyssynchronous behavior and reduced ejection fraction and were classified as a separate group from the controls and those AC-LV patients who did not have dyssynchrony impairement and/or LVEF and 2) radial dyssynchrony was the most affected parameter among the most affected AC-LV patients.

The clustering algorithm applied obtained a good cluster quality, with a silhouette value of 0.6 . It defined 2 clusters, in one of them all the controls were included, together with the AC-LV patients with normal dyssynchrony and LVEF parameters (cluster 1). In cluster 2, the AC-LV patients with dyssynchrony and LVEF impairement were included. Cluster 2 obtained much higher dyssynchrony values than cluster 1 (between 1.5 and 1.8 times higher in average) but those parameters were also more dispersed than in cluster 1 (higher standard deviation values). As expected, the LVEF was higher in cluster 1 than in cluster 2. 
The results found here are in line with our previous study, where we also found that radial dyssynchrony was the most discriminant parameter. In our previous work we found a threshold of $70 \mathrm{~ms}$ for radial dyssynchrony and $48.5 \%$ for LVEF that allowed to discriminate between controls and patients [8]. Similar values could be deduced from Table 1 of the present study.

This study presents some limitations. On the one side, the sample size is relatively small since AC-LV is a rare disease. On the other side, only one feature tracking package was available, thus further studies should be performed in order to ensure reproducibility.

\section{Conclusions}

Cine CMR and feature tracking allow the calculation of strain and dyssynchrony in the left ventricle. This study shows that $47 \%$ of the AC-LV patients present dyssynchrony impairment and decrease LVEF values. Radial dyssynchrony was the most discriminant parameter between normal and AC-LV most affected patients while longitudinal dyssynchrony was the less significant feature.

\section{Acknowledgments}

This work has been supported by the Spanish Government, under the grant DPI2015-70821-R received from the research program 'Retos de la Sociedad' by the Ministry of Economics and Competitiveness.

\section{References}

[1] Corrado D. Cardiomyopathy: Arrhythmogenic right ventricular cardiomyopathy: diagnosis, prognosis, and treatment. Heart. 2000 May 1;83(5):588-95.

[2] Sen-Chowdhry S, Syrris P, Prasad SK, Hughes SE, Merrifield R, Ward D, et al. Left-Dominant Arrhythmogenic Cardiomyopathy. Journal of the American College of Cardiology. 2008 Dec;52(25):2175-87.

[3] Marcus FI, McKenna WJ, Sherrill D, Basso C, Bauce B, Bluemke DA, et al. Diagnosis of arrhythmogenic right ventricular cardiomyopathy/dysplasia: Proposed Modification of the Task Force Criteria. European Heart Journal. 2010 Apr 1;31(7):806-14.

[4] Sreedevi BR, Usharani M. Comparative study of ejection fraction between middle aged and elderly males and females (age group 40-70 years). Journal of Evolution of Medical and Dental Sciences. 2015 Jun 9;4(47):8171-5.

[5] Onishi T, Saha SK, Ludwig DR, Onishi T, Marek JJ, Cavalcante JL, et al. Feature tracking measurement of dyssynchrony from cardiovascular magnetic resonance cine acquisitions: comparison with echocardiographic speckle tracking. Journal of Cardiovascular Magnetic Resonance. 2013;15(1):95

[6] Prati G, Vitrella G, Allocca G, Muser D, Buttignoni SC, Piccoli G, et al. Right Ventricular Strain and Dyssynchrony Assessment in Arrhythmogenic Right Ventricular Cardiomyopathy: Cardiac Magnetic Resonance Feature-
Tracking Study. Circ Cardiovasc Imaging. 2015 Nov;8(11):e003647; discussion e003647.

[7] Vigneault DM, te Riele ASJM, James CA, Zimmerman SL, Selwaness M, Murray B, et al. Right ventricular strain by MR quantitatively identifies regional dysfunction in patients with arrhythmogenic right ventricular cardiomyopathy: RV Strain in ARVC by MR Feature Tracking. Journal of Magnetic Resonance Imaging. 2016 May;43(5):1132-9.

[8] Vives-Gilabert Y, Sanz-Sánchez J, Molina P, Cebrián A, Igual $B$, Calvillo-Batllés $P$, et al. Left ventricular myocardial dysfunction in arrhythmogenic cardiomyopathy with left ventricular involvement: A door to improving diagnosis. International Journal of Cardiology [Internet]. 2018 Sep

[9] Theodoridis S, Koutroumbas K. Pattern recognition. 4. ed. Amsterdam: Elsevier Acad. Press; 2009. 961 p.

[10] Igual B, Zorio E, Maceira A, Estornell J, Lopez-Lereu MP, Monmeneu JV, et al. Resonancia magnética cardiaca en miocardiopatía arritmogénica. Tipos de afección y patrones de realce tardío de gadolinio. Revista Española de Cardiología. 2011 Dec;64(12):1114-22.

[11] Kern MJ. The cardiac catheterization handbook. Philadelphia, PA: Saunders Elsevier; 2011.

[12] Rokach L, Maimon O. Clustering Methods. In: Maimon O, Rokach L, editors. Data Mining and Knowledge Discovery Handbook [Internet]. Boston, MA: Springer US; 2005. p. 321-52. Available from: https://doi.org/10.1007/0-38725465-X_15

[13] Bacher J, Wenzig K, Vogler M. SPSS TwoStep Cluster - a first evaluation. Work and discussion paper. ErlangenNuremberg, Germany: Department of Sociology, Social Science Institute; 2004.

[14] Rousseeuw PJ. Silhouettes: A graphical aid to the interpretation and validation of cluster analysis. Journal of Computational and Applied Mathematics. 1987 Nov;20:5365 .

Address for correspondence:

Yolanda Vives Gilabert.

Instituto ITACA Edificio 8G

Universitat Politècnica de València

Camino de Vera s/n

46022, Valencia (Spain)

yovigi@teleco.upv.es 